\title{
HABERLAND SYNDROME
}

\author{
V. L. Ratnakumari¹, Vinoo Jacob², Parvathy S. Nair³, P. Y. Henry ${ }^{4}$
}

\section{HOW TO CITE THIS ARTICLE:}

V. L. Ratnakumari, Vinoo Jacob, Parvathy S. Nair, P. Y. Henry. "Haberland Syndrome". Journal of Evolution of Medical and Dental Sciences 2014; Vol. 3, Issue 35, Aug 14; Page: 9235-9240,

DOI: $10.14260 /$ jemds/2014/3191

ABSTRACT: Encephalo cranio cutaneous lipomatosis (ECCL) is a rare neuro-cutaneous syndrome. It is characterized by unilateral lipomas of the cranium, face, and neck, ipsilateral lipodermoids of the eye, ipsilateral brain anomalies. There are 53 cases mentioned so far in the literature. To our knowledge, only 3 cases were reported from India. We report a case of a baby girl who presented in our institution for neuro-radiological evaluation based on which diagnosis of ECCL was made.

KEYWORDS: Lipoma, lipodermoids, brain anomalies

INTRODUCTION: Encephalo cranio cutaneous lipomatosis (ECCL) or Haberland syndrome was first described by Haberland and Perou in 19701, 2. It is also referred as Fishman's syndrome. ECCL is a rare syndrome that is largely characterized by:

- Unilateral cyst with cortical atrophy

- Ipsilateral connective tissue, usually lipomatous hamartomas of the scalp, eyelid, and outer globe of the eye

- Cranial asymmetry

- Marked developmental delay and mental retardation

- Seizures

- Spasticity of the contralateral limbs

- Unilateral odontomas

\section{CASE REPORT:}

- A 3 month old girl born of non-consanguineous marriage, presented with a swelling of the scalp since birth.

- The swelling has not increased in size.

- The child also has multiple swellings on the right eyebrow, eyelids and sclera.

- No history of seizures or other neurological deficits.

- Mild developmental delay was noted.

\section{Physical Examination:}

- A 6x5 cm nontender, cystic swelling in the right frontal region of scalp.

- Focal alopecia noted over the swelling and over the frontal region [Fig.1].

- Multiple nodular lesions in the medial and lateral canthus of the right eye [Fig 2].

- A cleft/skin thickening running from the lateral side of right eye ending in front of the pinna [Fig.1].

- Brownish nodular lesion noted on the temporal side of the right eye.

- Coloboma of the right upper eyelid. 


\section{CASE REPORT}

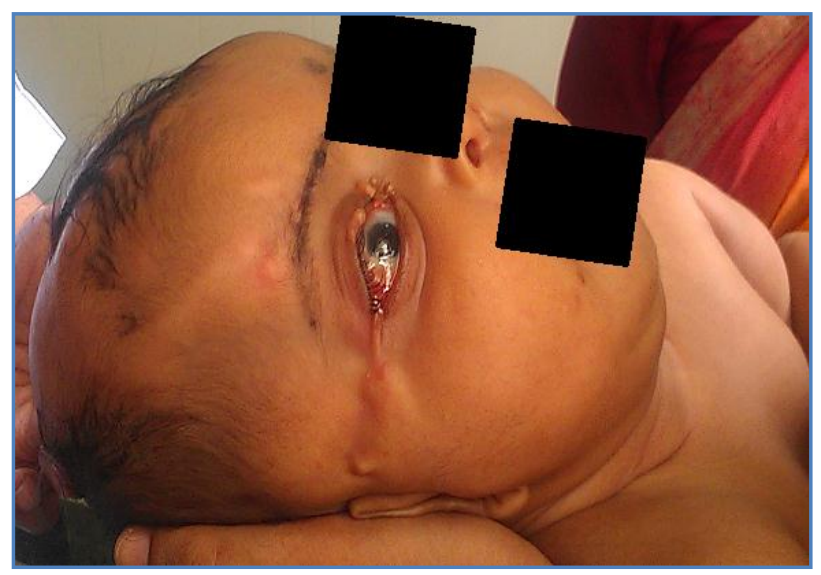

\section{Figure 1}

Fig. 1: A cleft/skin thickening running from the lateral side of right eye ending in front of the pinna and multiple nodular lesions in the medial and lateral canthus of right eye.

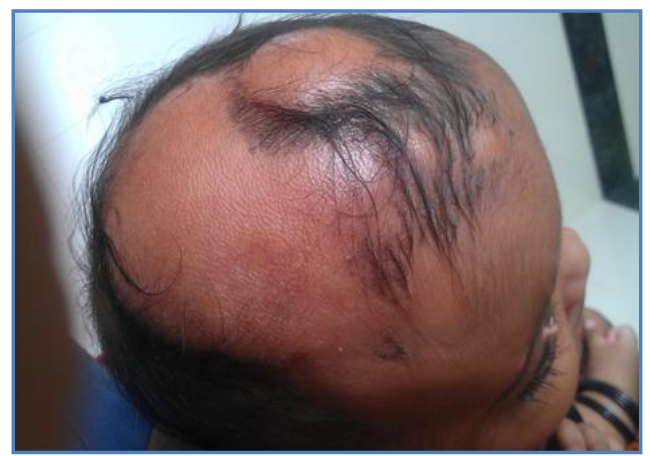

\section{Fig. 2: Focal alopecia noted over the swelling} and overthe frontal region

MRI of brain was done to evaluate the swelling. 


\section{CASE REPORT}
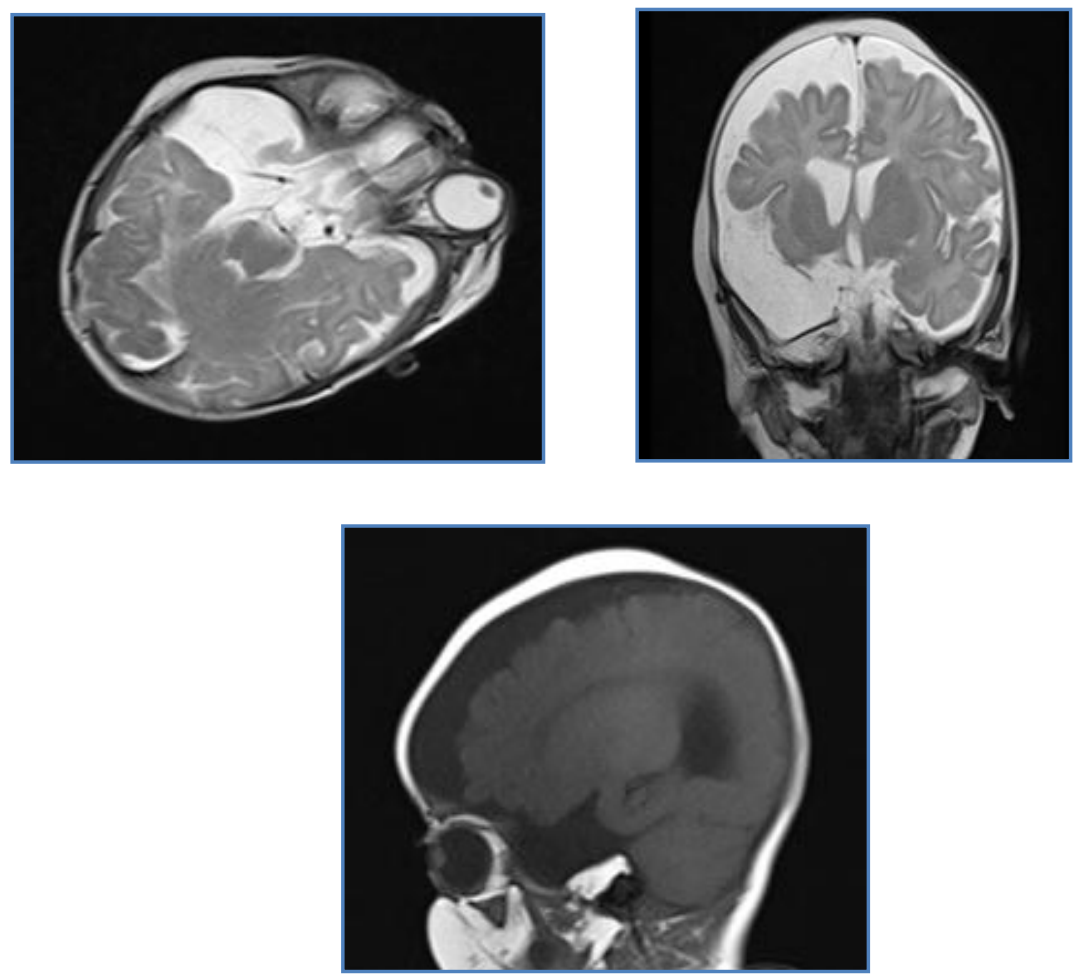

Figure 3

Fig. 3: T2 weighted MR image axial, coronal and T1 sagittal sections through temporal lobe showing increased extra axial space (suggestive of arachanoid cyst -T1 hypointense and T2 hyperintense signal) and poor insular operculisation on the right side.

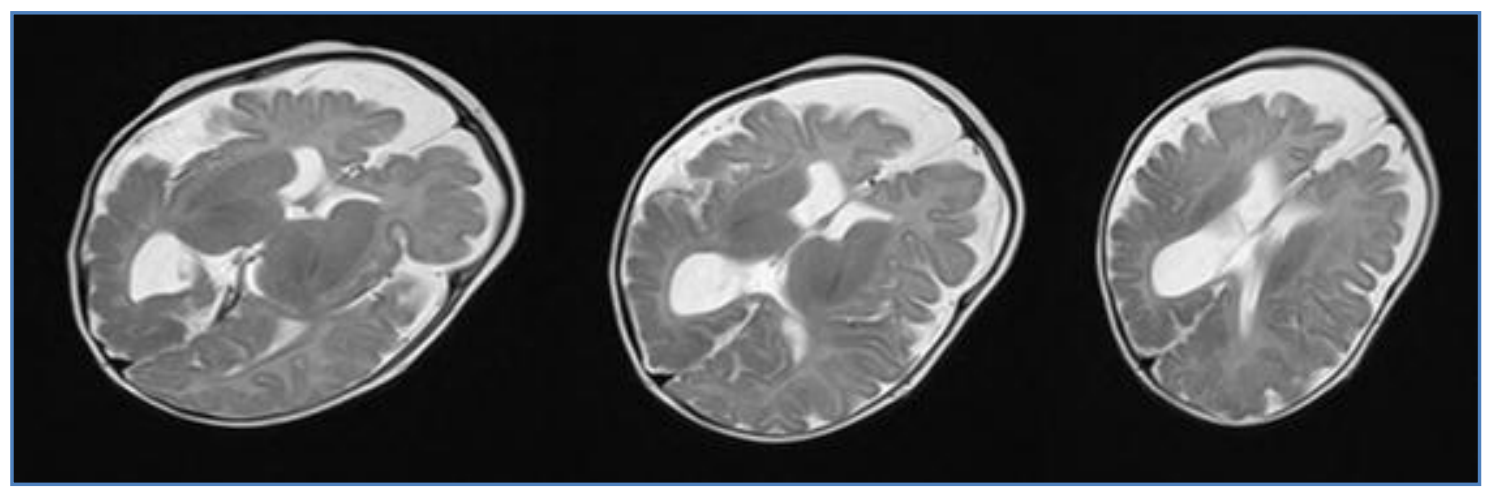

Figure 4

Fig.4: T2weighted MR axial image of brain showing increased extraxial space and dilated ventricles in the right temporal lobe suggestive of atrophy of the right hemisphere 


\section{CASE REPORT}
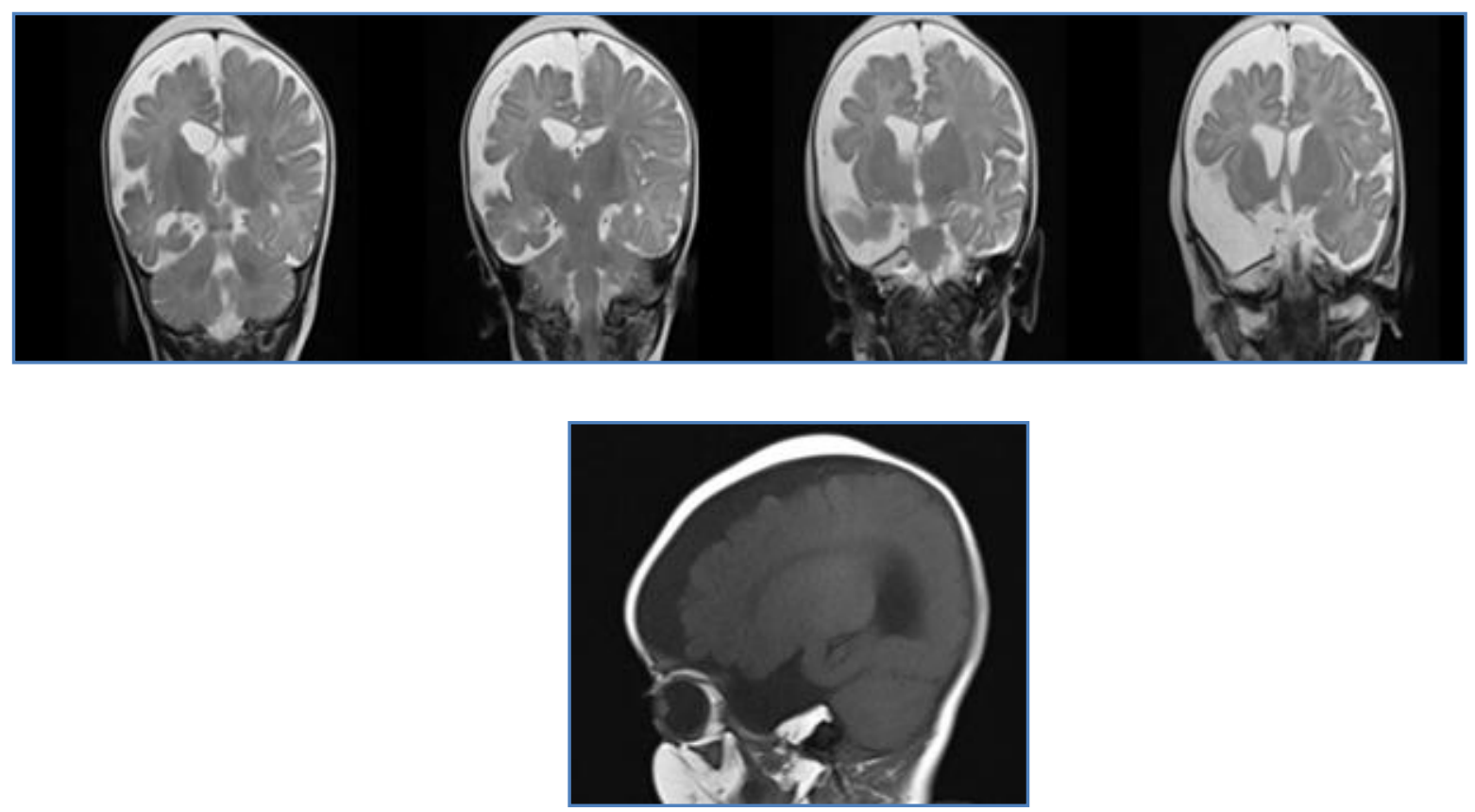

Figure 5 : Encephalo Cranio Cutaneous

Fig. 5:T2 weighted coronal and T1 weighted sagittal images showing an extra cranial scalp swelling suggestive of lipoma (T1 and T2 hyperintense).

Nodular skin lesions were biopsied, which came up as Lipomatous hamartomas. In the light of presenting features:

- Hairless lesion of the scalp suggestive of lipoma $a^{3-5}$

- Nodular skin lesions limited to, the right side of the face- Lipomas-8

- Hemiatrophy of brain, pooroperculisation of insular cortex and arachanoid cyst $^{9}$ on the same side of cutaneous lesions;

Diagnosis of EncephaloCranio Cutaneous Lipomatosis (ECCL) or Haberland Syndrome was made.

The child is under follow up at our institution.

\section{DISCUSSION:}

- Encephalo cutaneous lipomatosis (ECCL) is a rare neurocutaneous syndrome first described in 1970 by Haberland and Perou it is characterized by

- Unilateral lipomas of the cranium, face, and neck,

- ipsilateral lipodermoids of the eye.

- Ipsilateral brain anomalies.

- Pathogenesis is most likely a dysgenesis of the cephalic neural crest and anterior neural tube ${ }^{3}$

- ECCL is limited to one side of the cranium, the face, and brain. ${ }^{10}$

- The most typical lesions are subcutaneous soft tumors, consistent with lipomas, and areas of alopecia $^{5}$

- The hairless lesion of the scalp ("naevuspsiloliparus") is pathognomonic. 
- Other common findings are ocular lesions, such as defects of the eyelids and epibulbar dermoids. All these lesions seem to be nonprogressive.

- Multiple brain malformations on the same side as the head lesions are associated. Usually, seizures develop, beginning in infancy, and affected patients have variable degrees of psychomotor delay and motor impairment

The differential diagnosis includes $[9,11,12]$

- Sebaceous nevus syndrome,

- Oculocerebrocutaneous syndrome (OCC), and

- Proteus syndrome.

- The sebaceous nevus syndrome is associated with linear sebaceous nevi limited to one side of the face, ocular abnormalities, and clinical symptoms in common with ECCL. The most frequent CNS abnormality is widening of the ipsilateral ventricle.The sebaceous nevus syndrome and ECCL may thus be a continuum of phenotype expression

- OCC syndrome and ECCL share, as well, many clinical features. In OCC syndrome, the presence of orbital cyst, microphthalmia, and skin defects, as well as the absence of facial lipomas and scalp alopecia, are helpful for the diagnosis. In addition, CNS malformations, rare in OCC syndrome, are limited to intracranial cysts and agenesis of the corpus callosum.

- Proteus syndrome is an overgrowth syndrome in which every feature of ECCL can be found; it is bilateral, asymmetric, and involves the head, trunk, and limbs. Brain changes are rare. In contrast, ECCL is not progressive, unilateral, or limited to the head.

- The precise neuropathology of cortical dysgenesis cannot be based on neuroimaging findings; the histologic examination performed in one case revealed a polymicrogyric convolutional pattern.

- Intracranial lipomas were found in very few cases. In one, a leptomeningeallipo angiomatosis was also observed. 5

- A dysmorphic appearance of the corpus callosum, as well as unilateral ocular calcifications was found in two cases.

CONCLUSION: Even though rare, Haberland syndrome can be considered among the differentials in suspected cases of neurocutaneous syndrome.

\section{REFERENCES:}

1. Diagnostic Imaging: Paediatric Neuroradiology. Barkovich First Edition Page No. I-8-30.

2. Encephalo cranio cutaneous Lipomatosis: Complete Neuroradiologic Evaluation and Follow-up of Two Case Cecilia Parazzini et al AJNR 1999 20: 173-176

3. Hunter AG. Related Articles et al: Oculocere brocutaneous and encephalo cranio cutaneous slipomatosis syndromes: blind men and an elephant or separate syndromes? Am J Med Genet A. 2006; 140: 709-26.

4. Sofiatti A et al: Encephalo cranio cutaneous lipomatosis: clinical spectrum of systemic involvement. Pediatr Dermatol. 2006 Jan-Feb;23(1):27-30

5. Happle R et al: Nevus psiloliparus: report of two nonsyndromiccases. Eur J Dermatol. 2004; 14(5):314-6.

6. Gawel J et al: Encephalo cranio cutaneous lipomatosis. J Cutan Med Surg. 2003;7(1):61-5 
7. Rizzo R et al: Encephalo cranio cutaneous lipomatosis, Proteus syndrome, and somatic mosaicism.Am J Med Genet. 1993;47(5):653-5.

8. Fishman MA. Related Articles et al: Encephalo cranio cutaneous lipomatosis.J Child Neurol. 1987;2(3):186-93.

9. Nosti-Martinez D, Del Castillo V, Duran-Mckinster C, Tamayo-Sanchez L, Orozco-Covarrubias M, Ruiz-Maldonado R. Encephalo cranio cutaneous lipomatosis: an uncommon neurocutaneous syndrome. J Am AcadDermatol 1995; 32: 387-389.

10. Encephalo cranio cutaneous Lipomatosis (Haberland's Syndrome)-A Case Report of a Neurocutaneous Syndrome and a Review of the Literature Giovanna Negrisoli Koishi et al Clinics. 2008 June; 63(3): 406-408

11. Gokhale NR, Mahajan PM, Belgaumkar VA, Pradhan SN, Uttarwar NS. Encephalo cranio cutaneous lipomatosis: a rare neurocutaneous syndrome.Indian J DermatolVenereolLeprol. 2007; 73:40-2.

12. HaberlandC, Perou M. Encephalo cranio cutaneous lipomatosis: a new example of ectodermal dysgenesis.Arch Neurol. 1970; 22:144-55.

\section{AUTHORS:}

1. V. L. Ratnakumari

2. Vinoo Jacob

3. Parvathy S. Nair

4. P. Y. Henry

\section{PARTICULARS OF CONTRIBUTORS:}

1. Professor, Department of Radio diagnosis, Sree Gokulam Medical College and Research Foundation.

2. Professor, Department of Radio diagnosis, Sree Gokulam Medical College and Research Foundation.

3. Resident, Department of Radio diagnosis, Sree Gokulam Medical College and Research Foundation.
4. Professor, Department of Pediatric Surgery, Sree Gokulam Medical College and Research Foundation.

\section{NAME ADDRESS EMAIL ID OF THE} CORRESPONDING AUTHOR:

Dr. Vinoo Jacob,

Professor,

Sree Gokulam Medical College and Research Foundation, Venjaromoodu, Post Office, Thiruvananthapuram-695607.

E-mail: drvinoojacob@hotmail.com

Date of Submission: 12/02/2014.

Date of Peer Review: 13/02/2014.

Date of Acceptance: 13/03/2014.

Date of Publishing: 13/08/2014. 